\title{
Discrimination of People with Parkinson (PWP) Disease on the basis of Voice Parameter Analysis
}

\author{
Dixit \\ M.Tech. Student, \\ Department of ECE, Maharishi \\ Markandeshwar University, \\ Mullana, Haryana, India
}

\author{
Vikas Mittal \\ Asst. Professor, \\ Department of ECE, Maharishi \\ Markandeshwar University, \\ Mullana, Haryana, India
}

\author{
Yuvraj Sharma \\ Asst. Professor, \\ Department of ECE, Maharishi \\ Markandeshwar University, \\ Mullana, Haryana, India
}

\begin{abstract}
Voice is the essential medium of man's communication in social as well as professional interactions. The human voice also reflects the state of health in many medical conditions which leads voice alterations in patients. This paper presents a voice analysis approach for discriminating the People With Parkinson (PWP) on the basis of extracted voice parameters. Voice analysis basically deals with decomposition of voice signal into voice parameters for processing the resulted features in desirable application. The features that are extracted in this paper are: frequency, pitch, voice intensity, formant, speech rate and pulse functions like Jitter (local), Jitter (local, absolute), Jitter (rap), Jitter (ppq5), Jitter (ddp), Shimmer (local), Shimmer (local, dB), Shimmer (apq3), Shimmer (apq5), Shimmer (apq11), Shimmer (dda) and Harmonic coefficients.
\end{abstract}

\section{Keywords}

Voice analysis technique, PWP, prosody features, voice dysphonia, voice parameters, Hypokinetic dysarthria

\section{INTRODUCTION TO PARKINSON'S DISEASE (PD) AND ITS SYMPTOMS}

Parkinson's disease (PD) is one of the most pandemic neurodegenerative disorders that affect the control mechanism of nervous system of the human body. According to the Michael J. Fox Foundation for Parkinson's Research more than five million people in the worldwide, have Parkinson's disease and prevalence will double over the next 20 years. Parkinson's disease was named after an English doctor James Parkinson, who was first to characterize the disease extensively in 1817 [1] [3]. Parkinson's disease results from the loss of brain cells (neurons) mainly from a region of mesencephalon part of the brain called the substantia nigra along with the some components of the basal ganglia including the globus pallidus, putamen and caudate nucleus. The substantia nigra cells are responsible for producing a chemical substance known as dopamine, which act as a messenger for transmitting signals within the brain that allow for coordination of body movements. The shortage of the dopamine causes neurons to fire without normal control, leaving patients with the defects in motor as well as nonmotor body functions. The types of Parkinson's disease [4] [5] include Idiopathic Parkinson's disease (IPD), Parkinson-plus syndromes (PPS), and Symptomatic Parkinsonism. Idiopathic Parkinson's disease (IPD) is the predominant disorder, occurring in approximately $80 \%$ of diagnosed patients. Parkinson-plus syndromes (PPS) are the second most common types of the disorder and present in up to $15 \%$ of diagnosed patients. The early PD symptoms are non-motor symptoms which include disorders in the patient's voice (Dysphonia) which can cause neuropsychiatric problems like mood swings, cognition, behavior and thought alterations and sensory and sleep difficulties, followed by clinical motor symptoms [1] [2]. Motor symptoms in PD include tremor, slowness of movement, rigidity, and postural instability. The non-motor symptoms like irregularities in voice patterns i.e. Dysphonia symptoms can characterize Parkinson's disease in a subject [1]. The characteristic symptoms of Dysphonia in Parkinson's Disease is monotonous, low pitch or an inability to vary pitch and loudness, short speech, imprecise pronunciation, and a breathy and harsh voice [9]. The vocal impairment associated with PD is known as Hypokinetic dysarthria. Hypokinetic dysarthria can manifest in respiratory, phonatory, articulatory and resonatory subsystems of voice production, with the interplay of these subsystem impairments resulting in further disturbances in prosody and overall speech intelligibility [8]. At the respiratory level, some of the side effects that have been measured are: reduced vital capacity, lower rib cage volume, irregular breath patterns, reduced endurance of respiratory muscles, asynchrony of speech and respiration with chest wall movements during speech [6] [7]. Impairments at the articulatory level include reductions in: range, accuracy of articulatory movements, and tremor in the orofacial muscles [7] [8]. Features of phonatory hypofunction are: reduced vocal fold adduction; bowing; glottal chink; laryngeal phase asymmetry; and tremor [7]. PD is chronic as well as progressive i.e. it persists over a long period of time and its symptoms grow worse over time [13].

\section{VOICE PARAMETERS AS DISCRIMINATING FEATURE IN PWP}

Earlier, the detection of the onset symptoms of the Parkinson's disease was elusive because there was no objective test, or biomarker and that's why the rate of misdiagnosis can be relatively high. The difficulty in reliable PD diagnosis has inspired researchers to develop decision support tools relying on algorithms aiming to differentiate healthy peoples from people with Parkinson's (PWP). Research surveys conducted by the Parkinson's Disease Foundation till the year 2014 state that more than 90 percent of the people with Parkinson's experience changes in their speech. Persons with PD have characteristic slower speaking rates compared to normal speakers [10] [15]. Studies have found persons with PD produce fewer words, spend time producing speech per breath group, have more frequent pauses [17] [30] [16]. Voice Dysphonia also affects the phonatory system. The majority of persons with PD experience reduced vocal loudness. The vocal sound pressure level (SPL) in persons with PD has been shown to be statistically significantly lower during speech and 
voice tasks as compared to age-matched control subjects [19]. In later stages of PD voice of patients has diminished vocal loudness, with voices ranging from weakly to barely audible [14]. Persons with PD also exhibit difficulty achieving lowintensity phonation without going to the level of a whisper [21] [10], and they have a reduced ability to produce a loud voice when asked [10]. Laryngeal abnormalities in the form of bowed vocal cords and an abnormally large glottic aperture during phonation have been found to accompany those with PD [22] [23]. This results in incomplete approximation of the vocal cords, thus decreasing vocal loudness. Laryngeal dysfunction in the form of breathiness, hoarseness, roughness, tremor and abnormalities in vocal fold phase closure and phase symmetry were usually observed in persons with PD [24]. Reduced amplitude and firing rates of the major vocal fold adductor muscles, the thyroartenoid, [25] [26] contribute to reduced vocal loudness frequently accompanying PD. Buck and Cooper [20] stated that PD patients had equally affected phonation and articulation. Imprecise articulation is the impaired ability of persons with PD to perform diadochokinetic tasks such as specific lip movement, movement of tip, and back of the tongue required for $/ \mathrm{pa} /, / \mathrm{ta} /$, $/ \mathrm{ka} /$, and /pataka/ repetitions [11] [27] [28]. Vocal prosody can be defined as "the patterned distribution of stress, intonation, and other phonatory features of voice" [29]. Canter [9] [10] reported significant higher pitch levels and reduced pitch range for persons with PD. These variations may be dependent upon disease severity, co-existing conditions, and/or specific neurological substrate affected. There is a general belief that severity in voice increases with increased duration of PD. Another possible explanation for the hypo kinetic dysarthria variations and the lack of correspondence between limb and speech symptoms might be differences in the underlying neuropathophysiology of the disease across individuals and systems [31] [32]. Clearly, studies employing larger sample sizes with participants who exhibit a wide range of speech symptoms with well-controlled inclusion criteria are needed to aid in defining similarities in the patterns of voice. Several studies that have been performed investigating the patients of Parkinson's have concluded that PD patients tend to struggle with specific areas of prosody that are as follow:

- $\quad$ Parkinson's patients are often unable to produce the loud volume, pitch, voice modulations and rhythm patterns required for expressing certain emotions [12].

- Abnormal pauses in speech are also a characteristic of prosody associated with Parkinson, including pauses in common talk accompanied by pauses in between words or even in a single long word and speech rate also degrades [13].

Studies have also shown a progression of dysprosody in PD patients with time i.e. poor spoken skills, speech rate, and lowering of voice range with the progression of disease [13].

- $\quad$ PD patient exhibit reduced pitch, inability to vary pitch, short terms of speech continuity, imprecise consonants, inability to sustain prolonged vowel phonation. Their ability to sustain prolonged vowel phonation has been reported to be significantly impaired [10] [11].

Research studies have shown that speech may be a useful signal for discriminating PWP from healthy individuals, on the grounds of clinical evidence which imply that the vast majority of People With Parkinson (PWP) exhibit some form of vocal disorder and the fact that vocal impairment may be amongst the earliest appearing symptoms of Parkinson's Disease, can be detected even five years prior to clinical diagnosis [33]. These findings reinforce the fact that voice may reflect the disease status.

\section{ANALYSIS OF VOICE IN PWP}

Parkinson's disease is associated with the characteristic dysarthric voice patterns. Thus, analysis of vocal disorders can be an important medical procedure in diagnosing PD. This had inspired a great deal of research in biomedical engineering for developing clinical tools to evaluate pathological voice patterns. Besides, voice is a time-varying signal that contains all the useful information in its time variable characteristics. Voice analysis involves the transformation of voice signal into a set of parameters with an objective to simplify the voice signal to extract features directly pertinent for suitable applications. Thus, voice analysis tools allow the grasping of at least some of the distinguished time-varying features of voice, in order to ease the tasks of understanding, comparison, modification, and resynthesis. These Voice analysis tools are also used by speech pathologists and medical professionals to study, evaluate and diagnose the quality of the patient's voice signal on the basis of measurable features.

\subsection{PD Database and case studies accessed:}

The database employed for voice analysis has been selected to exhibit variable population patterns and diverse stages of disease. Table 1 illustrates database used for extracting specific features from voice samples. The voice samples as well as all the vital information required in the study are collected from the following resources:

- /VoiceMatters.net/, an online database of Dysarthic voice samples. This is a supporting community for patients and professionals who provide treatment or do research in the related areas.

- Harvard-Haskins database accessed at the link: /http://www.nsi.edu/ ani/speech_database.html/, maintained by the Neurosciences Institute (collected at Harvard University and Haskins Laboratories). The database contains acoustic data from 6 speakers (3 male, 3 female). All utterances in this database have 11 syllables, and consist of the syllable formed by vowels, numeric, long sentences, alternating with other syllables.

- NINDS Information sources accessed at the link: /http://www.nlm.nih.gov/medlineplus/parkinsonsdisease.html/ and an NIH online database that at /http://clinicaltrials.gov/, maintained by the National Institute of Neurological Disorders and Stroke (NINDS). The research on PD is one of the Institute's highest priorities. The Institute supports many scientist and research programs worldwide to achieve significant advances in halting the progression of PD, restoring lost function, and preventing the disease.

- American Speech-Language-Hearing Association hosts the information about the incidence and prevalence of dysarthria, its symptoms, evaluation, diagnosis and treatments at: /http://www.asha.org/public/speech/disorders/dysarthria/.

- $\quad$ The National Center for Voice and Speech (NCVS) hosts the link: /http://www.ncvs.org/research_parkinson.html/ for providing the latest information about voice and speech studies and researches on Parkinson disease and other neurological disorders.

- The Michael J. Fox Foundation for Parkinson's Research is one of the most credible organisations dedicated to finding a cure for Parkinson's disease through funded research agenda and ensuring the development of improved therapies for those living with PD. The Foundation assists the patient right from finding a doctor to life style, therapies and suitable treatments at the link: / https://www.michaeljfox.org/. 
Table 1: Database pattern used voice analysis.

\begin{tabular}{|l|l|l|}
\hline Subjects & Speech Utterances & Disease stage \\
\hline 5Females & Articulating sentences & Early stage \\
\hline 4 Females & Articulating sentences & Later stage \\
\hline 3 Males & Vowel Phonation & Early stage \\
\hline 6 Males & Spelling Phonation & Later stage \\
\hline 4 Females & Numeric phonation & Early stage \\
\hline 3 Males & Vowel Phonation & IPD recognition \\
\hline 5 Males & Vowel Phonation & Control subjects \\
\hline 5 Males & Articulating sentences & Control subjects \\
\hline 5 Males & Spelling Phonation & Control subjects \\
\hline
\end{tabular}

\subsection{Extracted Voice parameters from the Parkinson's subject and control samples:}

The significance of voice analysis is the extraction of voice features that contain all the auditory information as well as the spectral characteristics of the voice signal such as frequency, pitch, prosody etc. The extracted voice parameters from voice samples are:

Frequency: The frequency is extracted from the Spectrogram which provides a visual representation of voice in the form of the frequency variation with time of the resonance of a sound or series of sounds. Spectrograms are also called Voiceprints or Voicegrams. The spectrogram of a signal $\mathrm{s}(\mathrm{t})$ can be represented mathematically as the squared magnitude of the Short-Time Fourier Transform of the signal $\mathrm{s}(\mathrm{t})$, as: $\operatorname{Spectrogram}(t, w)=|\operatorname{STFT}(t, w)|^{2}$.

- Pitch: Pitch is defined as relative highness or lowness of voice. Pitch plots the perceived range of high versus low fundamental frequency of a person's voice. Pitch of the normal human voice is around the $2000-5000 \mathrm{~Hz}$. Fundamental frequency (F0) is mathematically stated as: $F O=1 / T, \mathrm{~T}$ is fundamental period given by: $\mathrm{x}(\mathrm{t})=\mathrm{x}(\mathrm{t}+\mathrm{T})$.

- Voice intensity: Vocal intensity is the major voice parameter, function of the amplitude of vibrations produced by vocal cord and pressure of the subglottic air stream. The dynamic range of voice intensity spans from $10^{-12} \mathrm{~W} / \mathrm{m}^{2}$ to 10 - $100 \mathrm{~W} / \mathrm{m}^{2}$. The Voice Intensity Level can be expressed mathematically as:

$$
L_{I}=10 \log \left(I / I_{\text {ref }}\right) \text {, }
$$

where $\mathrm{L}_{\mathrm{I}}=$ voice intensity level $(\mathrm{dB}), \mathrm{I}=$ voice intensity $\left(\mathrm{W} / \mathrm{m}^{2}\right), \mathrm{I}_{\mathrm{ref}}=10^{-12}$ is reference voice intensity $\left(\mathrm{W} / \mathrm{m}^{2}\right)$.

- $\quad$ Formant: Formants are defined as the spectral peaks of the sound spectrum of the voice. In speech science, formant is used to mean an acoustic resonance of the human vocal tract. A formant is a concentration of acoustic energy around a particular frequency in the voice signal. Formants exist in multiple values, each at a different frequency, roughly one in each $1000 \mathrm{~Hz}$ band. The formants arise from resonances produced in the vocal system of body. Thus, an individual can vary the vocal frequencies with the help of "articulator parts" resulting in changing the dimensions of the resonance cavities in the vocal tract. It is measured as peak amplitude in the frequency spectrum of voice given by coefficient of variation: $C V=\left[1 \ln \sum\left(x_{i}-\bar{x}\right)^{2}\right] / x^{-2}, \mathrm{n}$ is number of peaks measured, $x_{i}$ is individual amplitude value and $x$ is peak amplitude value.

Speech rate: The Speech rate measures the speed of speaking in words per unit interval. The normal averaging rate of speech in humans is about 100 to 125 words per minute.
Speech rate is part of the paralanguage of speech along with loudness and pitch. It conveys attitude and emotion.

- $\quad$ Pulse: The Pulse is an important feature for voice analysis which plots visible part of the sound wave. The Pulse shows multiple information about the voice measurements as: Jitter (local), Jitter (local, absolute), Jitter (rap), Jitter (ppq5), Jitter (ddp),Shimmer (local), Shimmer (local, dB), Shimmer (apq3), Shimmer (apq5), Shimmer (apq11), Shimmer (dda) and Harmonicity of the voiced parts.

- Jitter: Jitter defines variation in the pitch of voice. Jitter is the undesired deviation from true periodicity of a voice signal. Jitter may be observed in characteristics such as the frequency of successive pulses, the signal amplitude, of signals. Jitter is computed as the average absolute difference between the successive periods of fundamental frequency divided by the average period, computed in percentage as:

$$
\begin{aligned}
& N-1 \\
& \operatorname{Jitter}(\%)=\left[1 / N\left(\sum_{i=1}\left|T_{i}-T_{i+1}\right|\right)\right] /\left[1 / N\left(\underset{i=1}{\left.\left.\sum_{i} T_{i}\right)\right],}\right.\right.
\end{aligned}
$$

where $\mathrm{Ti}$ is the period of fundamental frequency of window number " $\mathrm{i}$ " and $\mathrm{N}$ is the total number of windows.

- Shimmer: Shimmer defines variation in loudness of voice. This is measured as the average absolute difference between the amplitudes of consecutive periods, divided by the average amplitude as:

$$
\text { Shimmer }=\left[1 / N-1\left(\sum_{i=1}^{N}\left|A_{i}-A_{i+1}\right|\right)\right] /\left[1 / N\left(\sum_{i=1}^{N} A_{i}\right)\right],
$$

where $A_{i}$ is the peak to peak amplitude of window number " $i$ " and $\mathrm{N}$ is the total number of windows. The Fig. 1 and Fig. 2 illustrate the visual representation of voice parameters in PRAAT window for PD samples and control samples respectively.

Fig. 1: The visual graphic presentation on PRAAT window

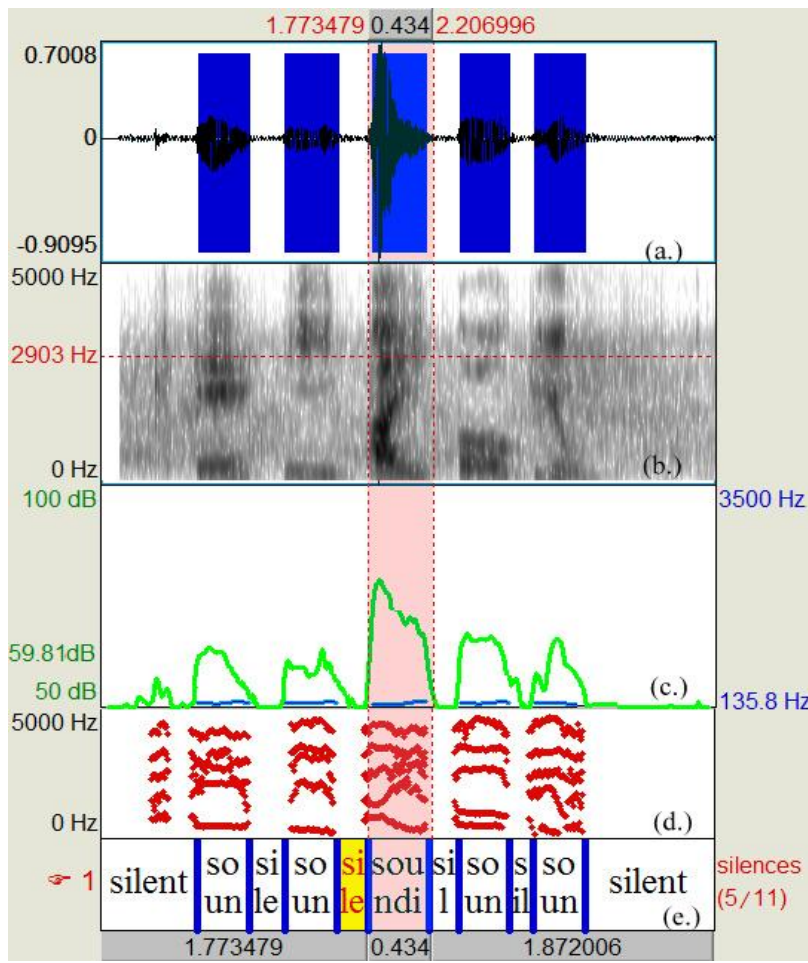

(a.) input voice sample, (b.) frequency spectrogram, (c.) pitch and vocal intensity, (d.) domain analysis of voice signal, (e.) speech rate of the PD samples. 


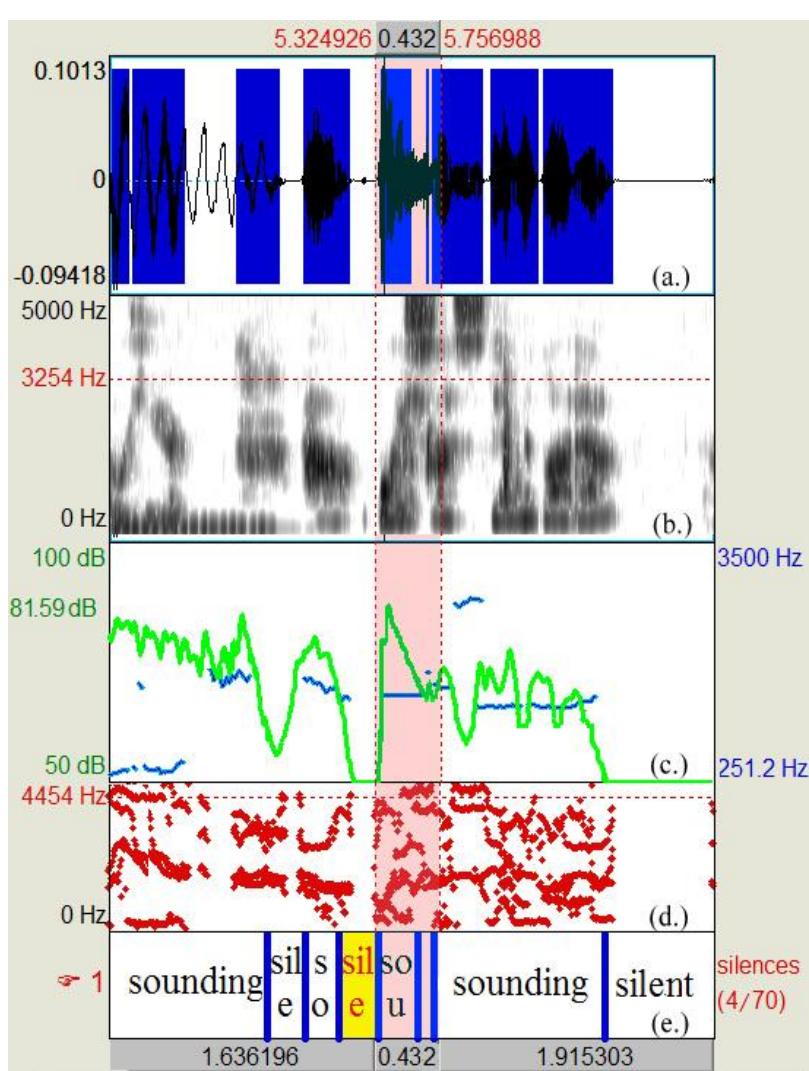

Fig. 2: The visual graphic presentation on PRAAT window (a.) input voice sample, (b.) frequency spectrogram, (c.) pitch and vocal intensity, (d.) domain analysis of voice signal, (e.) speech rate of the Control samples.

\subsection{Methodology and layout of work}

The objective of this study is to discriminate PWP on the basis of extracted voice features by analysis the voice signals. The software used is the PRAAT version 5.3.64 (2014) [34]. Praat is an efficient computer software package for the analysis of voice signal, developed by Paul Boersma and David Weenink on 1995 [34]. PRAAT is a very flexible tool that offers a wide range of standard and non-standard procedures, including spectrographic analysis, articulatory synthesis, and neural networks. This is helpful in clinical diagnosis of voice for acoustic evaluation of speech and voice samples. The work methodology involves the following steps in the order as stated below:

- $\quad$ The very first step of the research work is to build a database by collecting the voice samples exhibiting variable population patterns and diverse classes like as diseased subjects, controls, male, female, etc.

- This is followed by case studying the lifestyle, health background of subjects and collecting the information about the stage of the disease in subjects.

- Later the voice is input into the PRAAT tool installed in Windows7 operating system as wave file. PRAAT tool decomposes the voice signal into pulses of short time windows to extract the desired voice parameters of signal.

These parameters further yield the characteristic voice features which are as shown in Table 2 and Table 3 . Table 2 contains the extracted voice features of the PD subjects corresponding to the Visual graphical presentation of voice samples in Fig. 1 where as Table 3 represents the extracted voice features of the control subjects corresponding to the Visual graphical presentation of voice samples in Fig. 2.
The Fig. 3 summarizes the layout of all major steps involved in this research work to yield the objectives. The generated values corresponding to each extracted feature in both tables stood as base in distinguishing the People With Parkinson (PWP) from those of control subjects. Thus the voice parameters analysis on PRAAT platform has proven as an efficient tool discriminating the People With Parkinson (PWP) from control subjects and generated positive results.

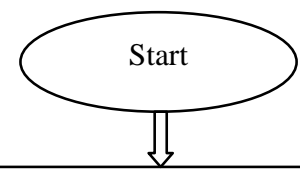

Collection of the voice samples and all PD related data.

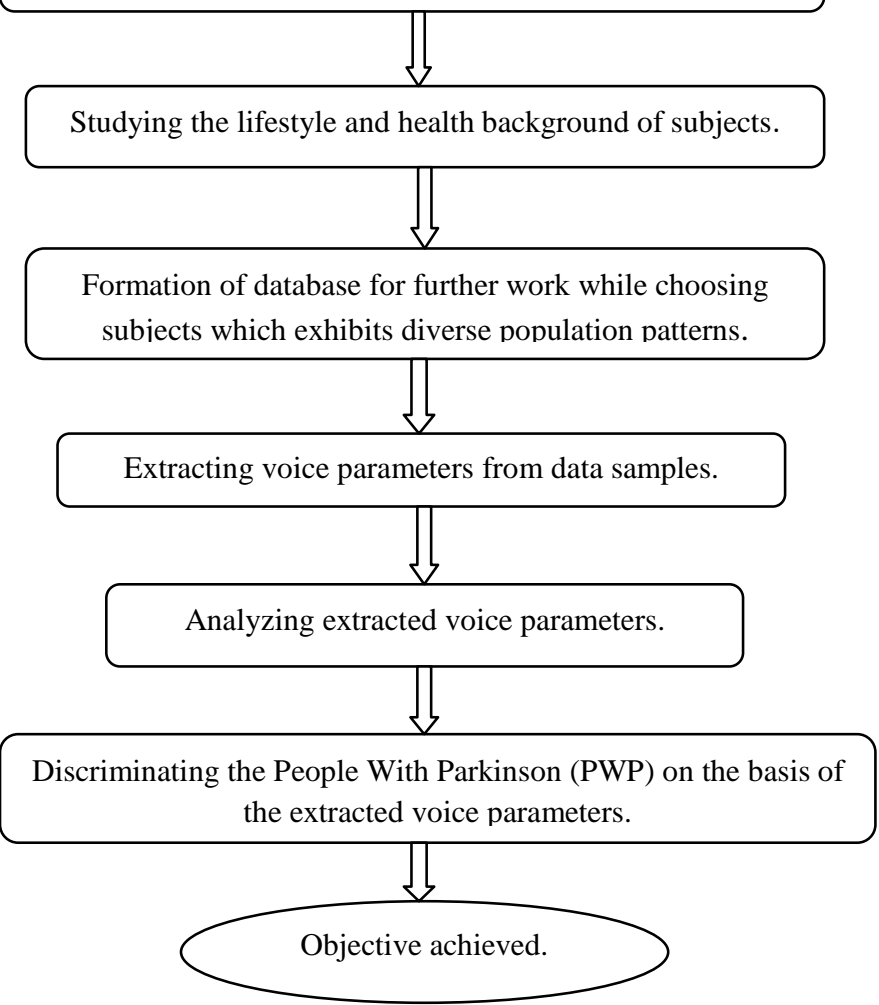

Fig. 3: Layout of the research work

\section{RESULTS AND DISCUSSION}

The plots obtained from the voice parameters analysis of voice samples succeed in discriminating PWP from control group. Table 2 illustrates the analysed extracted voice features of the PD subjects. Table 3 illustrates the extracted voice features of the control subjects.

Table 2: Extracted Voice features of PD subjects.

\begin{tabular}{|l|l|}
\hline Parameters & Extracted Features of PD subjects \\
\hline Time range & $\begin{array}{l}\text { From 1.773479 to 2.206996 seconds } \\
\text { (duration: 0.433517 seconds) }\end{array}$ \\
\hline Pitch & $\begin{array}{l}\text { Median pitch: } 128.091 \mathrm{~Hz} \\
\text { Mean pitch: } 135.782 \mathrm{~Hz} \\
\text { Standard deviation: } 16.079 \mathrm{~Hz} \\
\text { Minimum pitch: } 117.279 \mathrm{~Hz} \\
\text { Maximum pitch: } 168.229 \mathrm{~Hz}\end{array}$ \\
\hline Pulses & $\begin{array}{l}\text { Number of pulses: } 49 \\
\text { Number of periods: } 48 \\
\text { Mean period: } 7.393613 \mathrm{E}-3 \text { seconds }\end{array}$ \\
\hline
\end{tabular}




\begin{tabular}{|l|l|}
\hline Pulses & $\begin{array}{l}\text { Standard deviation of period: } \\
0.853359 \mathrm{E}-3 \text { seconds }\end{array}$ \\
\hline Voicing & Fraction of locally unvoiced frames: \\
& $\begin{array}{l}34.909 \% \text { (15/44) } \\
\text { Number of voice breaks: 2 } \\
\text { Degree of voice breaks:28.300\% } \\
\text { (0.122275 seconds / 0.432062 seconds) }\end{array}$ \\
\hline Jitter & Jitter (local): $2.224 \%$ \\
& Jitter(local,absolute):164.445E - 6 seconds \\
& Jitter (rap): 1.108\% \\
& Jitter (ppq5): 1.073\% \\
& Jitter (ddp): 3.325\% \\
\hline Shimmer & $\begin{array}{l}\text { Shimmer (local): } 14.835 \% \\
\text { Shimmer (local, dB): } 1.559 \mathrm{~dB}\end{array}$ \\
& $\begin{array}{l}\text { Shimmer (apq3): 5.712\% } \\
\text { Shimmer (apq5): } 8.325 \%\end{array}$ \\
& $\begin{array}{l}\text { Shimmer (apq11): } 15.067 \% \\
\text { Shimmer (dda): } 17.136 \%\end{array}$ \\
\hline $\begin{array}{l}\text { Harmonicity } \\
\text { of the voiced } \\
\text { parts }\end{array}$ & $\begin{array}{l}\text { Mean autocorrelation: } 0.856674 \\
\text { Mean noise-to-harmonics ratio: } 0.184752 \\
\text { Mean harmonics-to-noise ratio: } 8.709 \mathrm{~dB}\end{array}$ \\
\hline
\end{tabular}

Table 3: extracted voice features of Control subjects.

\begin{tabular}{|c|c|}
\hline Parameters & Extracted Features of Control subjects \\
\hline Time range & $\begin{array}{l}5.324926 \text { to } 5.756988 \text { seconds } \\
\text { (duration: } 0.432062 \text { seconds) }\end{array}$ \\
\hline Pitch & $\begin{array}{l}\text { Median pitch: } 230.776 \mathrm{~Hz} \\
\text { Mean pitch: } 251.198 \mathrm{~Hz} \\
\text { Standard deviation: } 61.258 \mathrm{~Hz} \\
\text { Minimum pitch: } 218.066 \mathrm{~Hz} \\
\text { Maximum pitch: } 456.348 \mathrm{~Hz} \\
\end{array}$ \\
\hline Pulses & $\begin{array}{l}\text { Number of pulses: } 71 \\
\text { Number of periods: } 68 \\
\text { Mean period: } 4.013338 \mathrm{E}-3 \text { seconds } \\
\text { Standard deviation of period: } \\
0.726909 \mathrm{E}-3 \text { seconds } \\
\end{array}$ \\
\hline Voicing & $\begin{array}{l}\text { Fraction of locally unvoiced frames: } \\
\text { Fraction of locally unvoiced frames: } \\
16.279 \% \text { ( } 7 \text { / 43) } \\
\text { Number of voice breaks: } 0 \\
\text { Degree of voice breaks: } 0 \\
(0 \text { seconds / } 0.433517 \text { seconds }) \\
\end{array}$ \\
\hline Jitter & $\begin{array}{l}\text { Jitter (local): } 3.132 \% \\
\text { Jitter (local, absolute): } 125.698 \text { E-6 seconds } \\
\text { Jitter (rap): } 1.452 \% \\
\text { Jitter (ppq5): } 1.512 \% \\
\text { Jitter (ddp): } 4.356 \%\end{array}$ \\
\hline Shimmer & $\begin{array}{l}\text { Shimmer (local): } 15.974 \% \\
\text { Shimmer (local, dB): } 1.487 \mathrm{~dB} \\
\text { Shimmer (apq3): } 7.283 \% \\
\text { Shimmer (apq5): } 10.777 \% \\
\text { Shimmer (apq11): } 16.007 \% \\
\text { Shimmer (dda): } 21.849 \%\end{array}$ \\
\hline $\begin{array}{l}\text { Harmonicity } \\
\text { of the voiced } \\
\text { parts }\end{array}$ & $\begin{array}{l}\text { Mean autocorrelation: } 0.839082 \\
\text { Mean noise-to-harmonics ratio: } 0.218698 \\
\text { Mean harmonics-to-noise ratio: } 8.546 \mathrm{~dB}\end{array}$ \\
\hline
\end{tabular}

\section{CONCLUSION}

The purpose of this research work was to state the applicability of voice parameter analysis in discriminating PWP on the basis of extracted voice features. The system employed for voice analysis has yielded better and positive outcomes. This application of voice analysis can be used for the clinical evaluation of PD patients. The features extracted are also represented in the form of mathematical functions for the suitability of the analysis for this application as described. This paper withstood the presence of irregularities in voice features as suggested by clinical case studies. The results obtain further verified the success of the voice analysis approach in the practical environments. Thus it can be concluded that the voice analysis approach can be a promising clinical diagnosis tool for such a fatal and incurable disease as Parkinson's Disease.

\section{ACKNOWLEDGMENTS}

The special thanks to NINDS (National Institute of Neurological Disorders and Stroke) Information office for proving Parkinson's data and information required for the research. The authors are thankful to Voice Matters.net for providing access to voice samples for the analysis and live patient interactions.

\section{REFERENCES}

[1] R. Arefi Shirvan, E. Tahami. "Voice Analysis for Detecting Parkinson's Disease Using Genetic Algorithm and KNN", IEEE Conference on BioMedical Engineering, 978-1-4673-1005-5/11, (December2011).

[2] Jankovic J. "Parkinson's disease: clinical features and diagnosis" ,J. Neurol. Neurosurg. Psychiatr. 79 (4): 368 76., (April 2008)

[3] E. Lang and A. M. Lozano, "Parkinson's disease", New England Journal of Medicine, vol. 339, no. 15, pp. 10441053, (1998).

[4] Dauer W., Przedborski, S., "Parkinson's Disease: Mechanisms and Models", Neuron, Cell Press, Vol. 39, 889-909, (September, 2003).

[5] Waters C.W., "Diagnosis and management of Parkinson's disease", NY: Professional Communications Vol. 4, (2005).

[6] Duffy J. R., "Motor speech disorders: Substrates, differential diagnosis, and management", 2nd ed., St. Louis, MO: Elsevier Mosby, (2005).

[7] Huber J. E., Stathopoulos E. T., Ramig L. O., Lancaster S. L., "Respiratory function and variability in individuals with Parkinson disease: Pre- and post-Lee Silverman Voice Treatment", Journal of Medical Speech-Language Pathology, 11(4), 185-201, (2003).

[8] Theodoros D. G., Murdoch B. E., Thompson E. C., "Hypernasality in Parkinson's disease: A perceptual and physiological analysis", Journal of Medical SpeechLanguage Pathology, 3(2), 73-84, (1995).

[9] Canter, G.J., "Speech characteristics of patients with Parkinson's disease:I. Intensity, pitch, and duration", Journal of Speech and Hearing Disorders, 28(3), 221229. (1963).

[10] Canter, G.J., "Speech characteristics of patients with Parkinson's disease: II. Physiological support for speech", Journal of Speech and Hearing Disorders, 30(1), 44-49. (1965a).

[11] Canter, G.J., "Speech characteristics of patients with Parkinson's disease: III. Articulation, diadochokinesis, and over-all speech adequacy", Journal of Speech and Hearing Disorders, 30(3), 217-224. (1965b). 
[12] Caekebeke, J F; Jennekens-Schinkel, A; Van Der Linden, $\mathrm{M}$ E; Buruma, O J; Roos, $\mathrm{R}$ A. "The interpretation of dysprosody in patients with Parkinson's disease". Journal of Neurology, Neurosurgery \& Psychiatry 54 (2): 145-148, (1991).

[13] Skodda, Sabine; Rinsche, Heiko; Schlegel, Uwe, "Progression of dysprosody in Parkinson's disease over time-A longitudinal study". Movement Disorders 24 (5): 716-22, (April 2009).

[14] Sidtis D.V.L., Rogers T., Godier V., Tagliat M., "Voice and Fluency Changes as a Function of Speech Task and Deep Brain Stimulation", American Journal of Speech, Language, and Hearing Research, Vol. 53, 1167-1177, (October 2010).

[15] Boshes, B., "Voice changes in Parkinsonism", Journal of Neurosurgery, 24, 286-290. (1966).

[16] Fox, C.M., \& Ramig, L.O., "Vocal sound pressure level and self-perception of speech and voice in men and women with idiopathic Parkinson disease", American Journal of Speech-Language Pathology, 6, 85-94. (1997).

[17] Hammen, V.L., \& Yorkston, K.M., "Speech and pause characteristics following speech rate reduction in hypokinetic dysarthria", Journal of Communication Disorders, 29, 429-444. (1996)

[18] Solomon, N.P., \& Hixon, T.J., "Speech breathing in Parkinson's disease", Journal of Speech and Hearing Research, 36, 294-310. (1993)

[19] Fox, C.M., \& Ramig, L.O., "Vocal sound pressure level and self-perception of speech and voice in men and women with idiopathic Parkinson disease", American Journal of Speech-Language Pathology, 6, 85-94. (1997).

[20] Buck, J.F., \& Cooper, I.S., "Speech problems in Parkinsonian patients undergoing anterior choroidal artery occlusion or chemopallidectomy", Journal of the American Geratric Society, 4, 1285-1290. (1956).

[21] Boshes, B., "Voice changes in Parkinsonism", Journal of Neurosurgery, 24, 286-290. (1966).

[22] Hanson, D., Gerratt, B., \& Ward, P., "Cinegraphic observations of laryngeal function in Parkinson's disease", Laryngoscope, 94, 348. (1984).

[23] Smith, M.E., Ramig, L.O., Dromey, C., Perez, K.S., \& Samandari, R., "Intensive voice treatment in Parkinson disease: Laryngostroboscopic findings," Journal of Voice, 9, 453-459. (1995).

[24] Perez, K.S., Ramig, L.O., Smith, M.E., \& Dromey, C., "The Parkinson larynx: Tremor and videostroboscopic findings", Journal of Voice, 10, 354-361. (1996).

[25] Baker, K.K., Ramig, L.O., Luschei, E.S., \& Smith, M.E., "Thyroarytenoid muscle activity associated with hypophonia in Parkinson disease and aging", Neurology, 51, 1592-1598. (1998).

[26] Luschei, E.S., Ramig, L.O., Baker, K.L., \& Smith, M.E., "Discharge characteristics of laryngeal single motor units during phonation in young and older adults and in persons with parkinson disease", Journal of Neurophysiology, 81, 2131-2139. (1999).

[27] Connor, N.P., Ludlow, C.L., \& Schulz, G.M., "Stop consonant production in isloated and repeated syllables in Parkinson's disease", Neuropsychologia, 27, 829-838. (1989).

[28] Hirose, H., Kiritani, S., Ushijima, T., Yoshioka, H., \& Sawashima, M., "Patterns of dysarthric movements in patients with Parkinsonism", Folia Phoniatrica, 33, 204 215. (1981).

[29] Scott, S., Caird, F.I., \& Williams, B.O., "Communication in Parkinson's Disease", Rockville, MD: Aspen. (1985).

[30] Metter, E.J., \& Hanson, W.R., "Clinical and acoustical variability in hypokinetic dysarthria", Journal of Communication Disorders, 19, 347-366. (1986).

[31] Alexander, G., Crutcher, M., \& DeLong, M., "Basal ganglia-thalamocortical circuits: Parallel substrates for motor, oculomotor, prefrontal and limbic functions," Progress in Brain Research, 85, 119-146. (1990).

[32] M. A. Little, P. E. McSharry, E. J. Hunter, J. Spielman, and L. O. Ramig, "Suitability of dysphonia measurements for telemonitoring of Parkinson's disease," IEEE Trans. Biomed. Eng., vol. 56, no. 4, pp. 1015-1022, (Apr. 2009)

[33] LeWitt P. A., "Parkinson's disease: Etiologic considerations", Parkinson's disease and movement disorders: Diagnosis and treatment guidelines for the practicing physician in C. H. Adler \& J. E. Ahlskog (Eds.), pp. 91-101, Totowa, NJ: Humana Press, (2000).

[34] Boersma P., Weenink D., "PRAAT: Doing phonetics by computer", Version 5.3.64, Computer program, 2014 